\title{
Oxidation of Nickel Particles in an Environmental TEM
}

Q. Jeangros ${ }^{1}$, T.W. Hansen ${ }^{2}$, J.B. Wagner ${ }^{2}$, R.E. Dunin-Borkowski ${ }^{3}$, C. Hébert ${ }^{1}$, J. Van herle $^{4}$ and A. Hessler-Wyser ${ }^{1}$

${ }^{1}$ Interdisciplinary Centre for Electron Microscopy, Ecole Polytechnique Fédérale de Lausanne, Lausanne, Switzerland

${ }^{2}$ Center for Electron Nanoscopy, Technical University of Denmark, Lyngby, Denmark

${ }^{3}$ Ernst Ruska-Centre for Microscopy and Spectroscopy with Electrons and Peter Grünberg Institute, Jülich Research Centre, Jülich, Germany

${ }^{4}$ Laboratory for Industrial Energy Systems, Ecole Polytechnique Fédérale de Lausanne, Lausanne, Switzerland

The mechanisms controlling the growth of an oxide film during oxidation are subject to controversies at intermediate length scales $(20-1000 \mathrm{~nm})$ [1]. Relating rate-controlling mechanisms and resulting structural changes, which is essential to the understanding of oxidation processes, has proved challenging under these conditions.

Here, nickel particles are oxidized under 3.2 mbar of $\mathrm{O}_{2}$ inside an environmental TEM (ETEM) equipped with a post-column filter [2]. Images, diffraction patterns and core-loss electron energy-loss spectra are acquired to monitor the structural and chemical evolution of Ni during oxidation, whilst increasing the temperature up to $600{ }^{\circ} \mathrm{C}$.

Nucleation of $\mathrm{NiO}$ on $\mathrm{Ni}$ is observed to occur rapidly at room temperature before the introduction of $\mathrm{O}_{2}$ in the environmental cell (in the vacuum of the microscope). It involves the formation of randomly orientated oxide domains of a few nanometres in size. These domains impinge and cover the particles surface. As the temperature increases under $\mathrm{O}_{2}$, the $\mathrm{NiO}$ film grows and creates irregular structures composed of many crystallites. The reaction kinetics are inferred by EELS using different techniques analyzing changes in shapes of the $\mathrm{Ni}_{2,3}$ white lines [3]. The results indicate that the oxidation process is diffusion-controlled, similarly to results from the literature that were obtained at larger oxide thicknesses [1]. Pores are observed to form at the $\mathrm{Ni} / \mathrm{NiO}$ interfaces, resulting in the loss of metal/oxide contact (Fig. 1). These observations illustrate that the outward diffusion of $\mathrm{Ni}^{2+}$ ions through $\mathrm{NiO}$ is the dominant mass transport mechanism under these conditions (in opposition to $\mathrm{O}_{2} / \mathrm{O}^{2-}$ transport). Images also indicate that the $\mathrm{NiO}$ film might rupture in some regions, a process that should enable some inward diffusion of $\mathrm{O}_{2}$ and therefore inward growth of $\mathrm{NiO}$. An activation energy for $\mathrm{Ni}$ oxidation comparable to the ones found in the literature is determined from our EELS data.

By using ETEM, we are able to relate the structural and chemical changes occurring at the nanoscale during the oxidation of $\mathrm{Ni}$ particles with $\mathrm{O}_{2}$ at high temperature, providing new insights into oxidation/corrosion processes.

[1] A. Atkinson, Reviews of Modern Physics, 57 (1985), p. 437.

[2] T.W. Hansen, et al., Materials Science and Technology, 26 (2010), p. 1338.

[3] Q. Jeangros, et al., Acta Materialia, 58 (2010), p. 4578.

[4] Support from the Swiss National Science Foundation is gratefully acknowledged (project "IN SItu TEm study of reduction and reoxidation of $\mathrm{Ni}(\mathrm{O})$-ceramic composite (INSITE)"). 

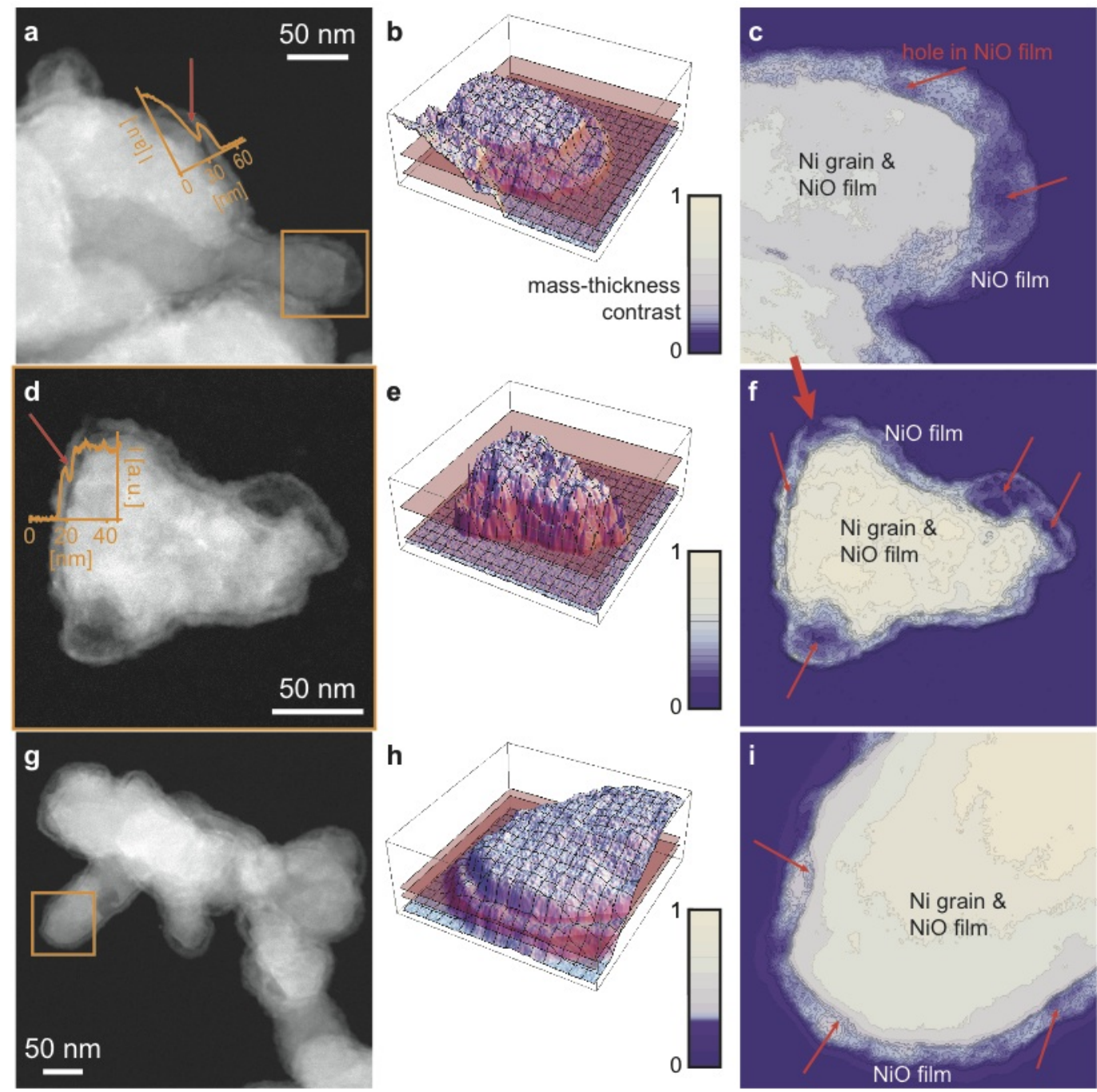

Figure 1: a, $\mathbf{d}$ and $\mathbf{g}$ STEM-HAADF images of $\mathrm{Ni}(\mathrm{O})$ particles at $300{ }^{\circ} \mathrm{C}$ under $3.2 \mathrm{mbar}$ of $\mathrm{O}_{2}$ along with intensity profiles. Arrows highlight a decrease in intensity at the $\mathrm{NiO} / \mathrm{Ni}$ interfaces. Corresponding 3D histograms and contour plots are shown in $\mathbf{b}, \mathbf{c}, \mathbf{e}, \mathbf{f}, \mathbf{h}$ and $\mathbf{i}$. The intensity scale of $\mathbf{c}, \mathbf{f}$ and $\mathbf{i}$ has been adapted for each image to illustrate the differences in contrast located between the planes marked in red on the histograms $\mathbf{b}, \mathbf{e}$ and $\mathbf{h}$. Arrows indicate holes in the NiO film, while the larger one in $\mathbf{f}$ indicates what seems to be a fracture in the $\mathrm{NiO}$ film. 